\title{
The role of Kifc1, Kchn5 and miRNA-302 on in vitro development in 8-cell, morula and blastocyst stage of mouse embryos
}

\author{
Konstantinos Ntzeros ${ }^{1}$, Despoina Mavrogianni ${ }^{2}$ Athina Koutsi ${ }^{2}$, Antonia Kandaraki ${ }^{2}$, \\ Anastasios Papadelas ${ }^{2}$, Sofoklis Stavros ${ }^{1}$, Petros Drakakis' ${ }^{1}$, Dimitrios Loutradis ${ }^{1}$
}

11st Department of Obstetrics \& Gynecology, "ALEXANDRA” General Hospital, Medical School of National and Kapodistrian University of Athens, Athens, Greece ${ }^{2}$ Molecular Biology Laboratory, IVF Unit, 1st Department of Obstetrics \& Gynecology, "ALEXANDRA" General Hospital, Medical School of National and Kapodistrian University of Athens, Athens, Greece

Correspondence

Loutradis Dimitrios, Alexandra Hospital, 80 Vasilissis Sofias Avenue, GR 115 28, Athens, Greece, email: loutradi@otenet.gr

\section{Abstract}

Introduction: Embryo development is characterized by lack of cell cycle check-points and overexpression of core circadian oscillators. On previous report we have identified several genes over-and under-detected at human embryo blastomeres. In this study, we investigated the expression profile of Kcnh5, KIFC1 and miRNA-302 genes at three pre-implantation stages of mouse embryo development. Material and methods: Total RNA was extracted from mouse embyos at 8-cell, morula and blastocyst stage. The expression profile of Kcnh5, KIFC1 and miRNA-302 was assessed by RT-PCR and the results were normalized with G6pdh expression levels. Results: Kcnh5 showed absence of expression at all stages, indicating novel mechanisms of cell cycle control during blastomeres divisions. KIFC1 showed positive expression at all stages, with decreasing levels as the embryogenesis progresses. This finding indicates that KIFC1 may have more important role at early events. miRNA-302 showed increased levels of expression at all stages, with morula having the highest levels. Therefore, miRNA-302 might play an important role at the events that happen during morula stage such as compaction. Conclusions: Cell cycle control of blastomeres at early embryogenesis might be based on different mechanisms compared to somatic cells and more research is needed in order to reveal crucial cycling elements.

Key words: Mouse embryo development, Kchn5, KIFC1, miRNA-302, cell cycle control, gene expression

\section{Introduction}

Genetic markers of the pre-implantation embryo are a challenging field concerning the quality of em- bryos. The aim is to achieve higher pregnancy rates, reduce multiple pregnancies and obtain healthier babies. To accomplish this purpose, it is crucial to de- 
fine the ability of oocytes to complete meiosis and undergo fertilization in order to produce a healthy embryo which has the potential to progress to the blastocyst stage in vitro and implant to produce healthy offspring. The selection of embryos with higher implantation potential is one of the major challenges in ART. Even though nowadays the majority of scientists use morphological criteria for the evaluation of embryos quality, we need strategies to implement the molecular technology in order to investigate the oocytes and embryos quality, regarding gene's expression ${ }^{1}$. Focusing on identifying the expression profile of each stage could be useful to unravel the mystery of these developmental stages ${ }^{2,3}$. Further understanding of the biological role of expressed genes may expand our knowledge for the oocyte maturation, fertilization and early embryogenesis steps. The practical implications of compiling gene expression information on human oocytes and embryos would be enormous since it could potentially help us to understand and solve problems related to quality of embryos and we will use the genetic profile as a non-invasive procedure of embryos evaluation. The aim of several investigators is the expression analysis and the investigation of the molecular profile across all stages of embryos development, as well as the function of known genes.

We have previously reported that the normal human 8-cell embryo is characterised by lack of cell cycle check-points and overexpression of core circadian oscillators and cell cycle drivers ${ }^{2,3}$. More specifically, circadian molecular components such as CLOCK, PERIOD, CRYPTOCHROME and ARNTL (BMAL) as well as Cyclins A, B, E and MYC of the cell cycle machinery are overexpressed. On the contrary, key cell cycle checkpoints $\mathrm{Rb}$ and Wee1 are underexpressed ${ }^{2,3}$. We also examined the expression profile of growth factor families (Tgfb, Fgf, Egf) and cytokines in 8-cell embryos, finding a circadian rhythm-independent modulation of such genes in early embryogenesis ${ }^{4}$.

Additionally, we identified new molecules that participate in early embryonic divisions such as Kcnh5 (Eag2), a voltage-gated potassium channel and KIFC1, a kinesin family member, which were overexpressed at 8-cell human embryos ${ }^{3}$. Kcnh5 belongs to the ether-a-go-go (eag) potassium channel family and is associated with cell cycle progression via regulating cell volume dynamics ${ }^{5}$. Kcnh 5 was also shown to bind to alpha and beta-tubulin, indicating an important role in the cytoskeleton formation at early embryogenesis ${ }^{7}$ Knockdown of Kcnh5 resulted in inhibition of proliferation, arrest of cells at G1 phase or even triggered apoptosis depending on cell type, indicating a crucial role in proliferation ${ }^{3,4}$. Another important observation is that Kcnh5 shows low levels of expression in human oocytes following upregulation after fertilization ${ }^{7}$.

KIFC1 is a member of Kinecin-14 family which is characterised by minus-end motility on microtubules ${ }^{8}$. This kinesin is associated with elongation and stabilization of spindle at mitosis and meiosis, biogenesis of acrosome during spermatogenesis and survival of cancer cells with extra centrosomes ${ }^{9-11}$. Additionally, KIFC1 was found overexpressed in syncytiotrophoblast of normal and pathological human placenta, indicating an active role during implantation $^{12}$.

MicroRNAs (miRNAs) are well-established geneexpression regulators which participate in cell cycle and developmental processes. The miRNA-302/367 cluster generally consists of five members, miRNA302a, miRNA-302b, miRNA-302c, miRNA-302d and miRNA-367 and it is ubiquitously distributed in vertebrates, considered an important regulator of cell cycle and embryonic development. More specifically, the miRNA-302/367 cluster is shown to have crucial role in G1/S transition at mouse embryonic stem cells, targeting cyclins and kinases, in order to promote proliferation ${ }^{13,14}$. Additionally, it participates in 


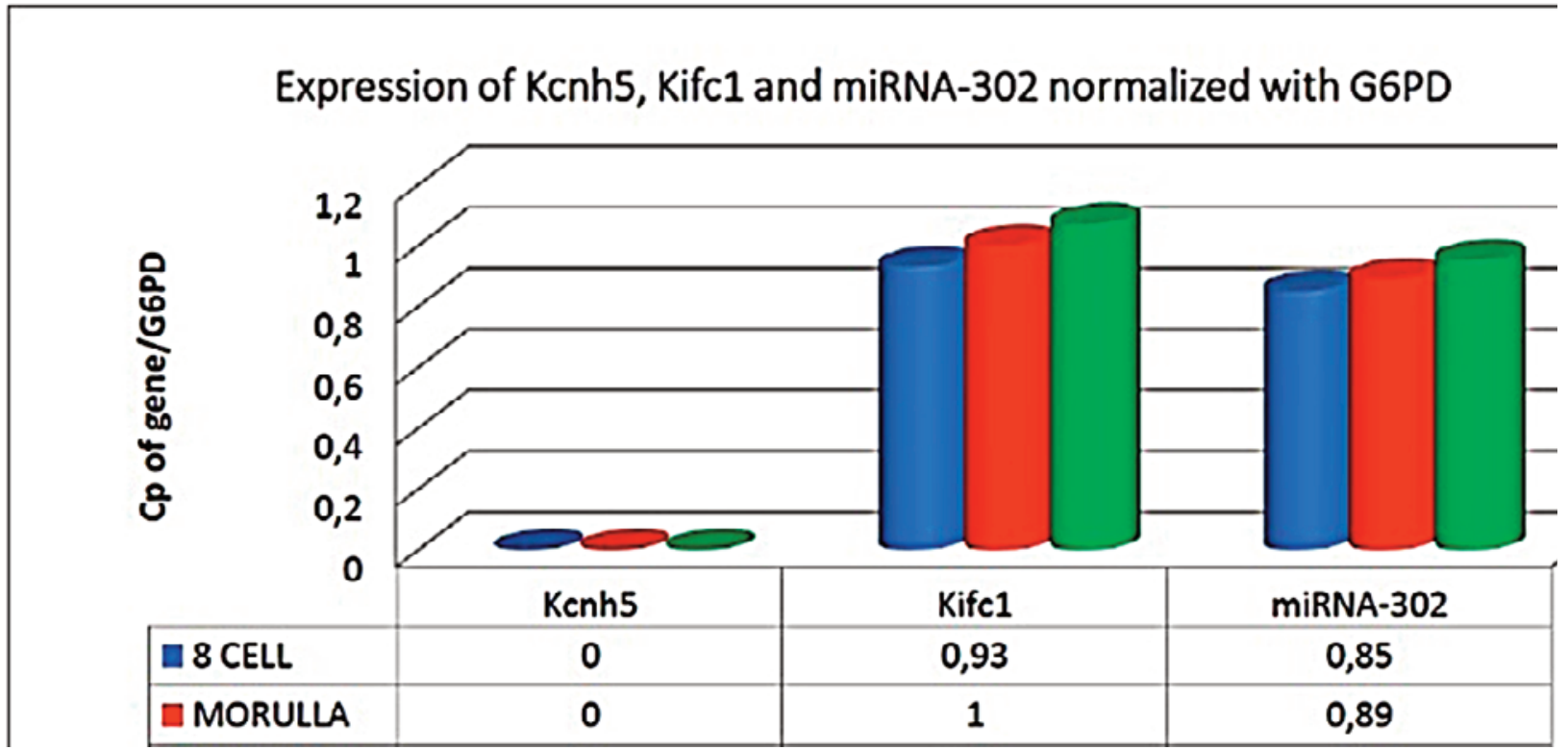

Figure 1. Expression of Kcnh5, Kifc1 and miRNA-302 normalized with G6PD at three developmental stages of mouse embryo, expressed as crossing point (Cp) ratio.

neurulation by suppressing differentiation and neural progenitor expansion ${ }^{15}$. miRNA-302 is also associated with somatic cell reprogramming through global demethylation, indicating a possible role in epigenetic reprogramming of the zygote as well ${ }^{16}$. However, there are conflicting data concerning the expression profile of miRNA-302/367 cluster at embryos. Viswanathan et al found insignificant expression levels of miRNA-302 family during early embryogenesis while Card et al showed that miRNA302 is expressed at developmental stages E6.5, E7.5 and $\mathrm{E} 8.5^{17,18}$.

On previous report, we have identified 35 cell cycle genes over-detected on whole human genome microarrays of 8-cell stage blastomeres and ten genes under-detected on 8-Cell arrays ${ }^{2}$. Therefore, the objective of this study is to investigate the expression profile of Kcnh5, KIFC1 and miRNA-302 at three pre-implantation stages of mouse embryo development (8-cell, morula and blastocyst). The relative ex- pression of these genes in mouse embryos will reveal which cycling elements are the most crucial for development.

\section{Materials and Methods}

\section{Embryo collection and Culture}

F1 (C57BL6 x CBA) females 3-4 week-old, were superovulated by 5 IU PMSG (Sigma Chemical Co) followed by 5 IU of hCG (Sigma Chemical Co) 48 hours later. Females were housed individually overnight with males F1 9-week-old and sacrificed 20 hours after hCG administration. Subsequently the oviducts, containing the fertilized oocytes, were removed and cultured at DPBS (Dulbecco's Phosphate Buffered Salin, Gibco) culture medium with 10\% BSA (SigmaAldrich). The oviducts were incised and the fertilized oocytes were removed, incubated with hyaluronidase, separated from cumulus cells and cultured at $1 \mathrm{ml}$ Ham's (Ham's F10 Medium (-) Hypoxanthine, Gibco) 
culture medium with $5 \mathrm{mg} / \mathrm{ml} \mathrm{BSA}$. The embryos, at groups of forty, were incubated $(37 \mathrm{oC}, 5 \% \mathrm{CO} 2,95 \%$ humidity) for 48 (8-cell stage), 72 (morula stage) and 96 hours (blastocyst stage).

All female and male mice used in this study were C57BL/6 female x CBA male F1 hybrids raised and cared for at the Pasteur Institute (Athens, Greece).

\section{RNA and cDNA preparation}

Total RNA was extracted from embryos of 8-cell, morula and blastocyst stage. Total RNA extraction kit was obtained from Qiagen (RNeasy micro kit, Qiagen) and the extraction was performed according to manufacturer's protocol. RNA concentration of each sample was determined by spectrophotometry and its quality was evaluated by agarose gel electrophoresis.

Twenty nanograms of total RNA were used for first-strand cDNA synthesis using random hexamer primers and Superscript II Reverse Transcriptase (Superscript II First Stand Synthesis System for RTPCR, Invitrogen) according to the manufacturer's protocol. The resulting cDNAs were stored at $-20^{\circ} \mathrm{C}$.

\section{Real-time PCR}

The expression of Kcnh5, KIFC1 and miRNA-302 gene were assessed at three pre-implantation stages of mouse embryo development (8-cell, morula and blastocyst) by real-time RT-PCR using primers and probes pairs particularly synthesized by TIB-MOLBIOL (Berlin, Germany) for this study (Table 1).

The specific primers and probes were used at a concentration of $20 \mathrm{pmol} / \mu \mathrm{l}$ in each reaction. Quantitative real-time PCR was performed in a final reaction volume of $20 \mu \mathrm{l}$ in a LightCycler 480 white 96-multiwell plate (Roche Diagnostics, Mannhein, Germany). All samples were run in duplicate, and notemplate controls were included in all runs to exclude possible DNA contamination. The RT-PCR mixture for Kcnh5 and KIFC1 contained 5x LightCycler 480 Genotyping Master (Roche), $0.5 \mu \mathrm{l}$ for each primer, $0.2 \mu \mathrm{l}$ for each probe, up to $20 \mu \mathrm{l}$ total volume of reaction $\mathrm{H}_{2} \mathrm{O}$ of LightCycler 480 Genotyping Master (Roche), and $5 \mu \mathrm{l}$ cDNA. The RT-PCR mixture for miRNA-302 contained 2x QuantiTect SYBR Green Master Mix (Qiagen), $0,4 \mu \mathrm{l}$ for each primer, up to $20 \mu$ l total volume

\section{Expression of Kcnh5, Kifc1 and miRNA-302 normalized with G6PD}

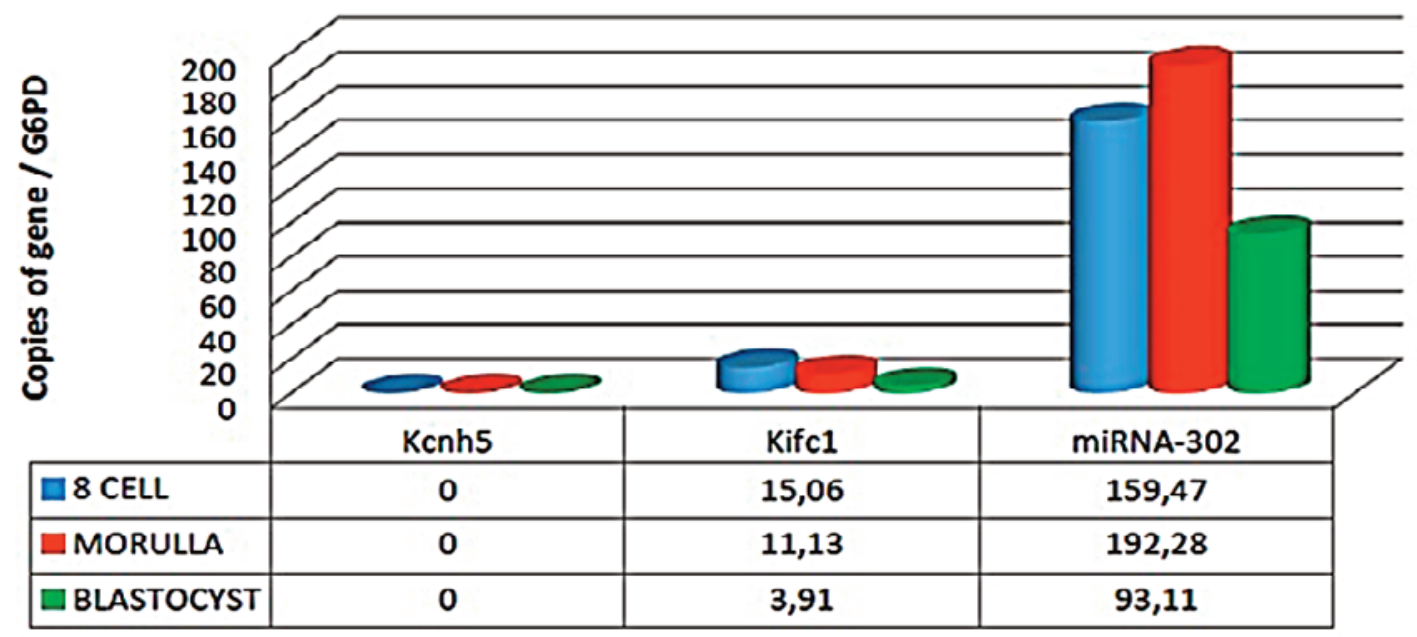

Figure 2. Expression of Kcnh5, Kifc1 and miRNA-302 normalized with G6PD at three developmental stages of mouse embryo, expressed as copies of gene ratio. 
Table I. Primers sense and antisense and probes fluorescein and LightCycler of KCNH5, KIFC1, INCENP, miRNA-302 and muG6PD

$\begin{array}{ll}\text { Gene } & \text { Sequence } \\ \text { KCHN5 } & \\ \text { KCNH5 S } & \text { ATAGGATTTGGAAACATAGC } \\ \text { KCNH5 A } & \text { TAACGGTTGGTGTTGGCA } \\ \text { KCNH5 FL } & \text { AAAGAAGAGCCGACCATCATCATG-FL } \\ \text { KCNH5 LC } & \text { 640-CCACTGAGAACATCTTCTCCACGTCTGT p } \\ \text { KIFC1 } & \\ \text { KIFC1 S } & \text { GGCGGGTGTGGCTGATTG } \\ \text { KIFC1 A } & \text { CCTCTTGAGCCTGCTTGCTGAC } \\ \text { KIFC1 FL } & \text { ACTTCACCAGGGCTGCCTTCAGTTC-FL } \\ \text { KIFC1 LC } & \text { 640-ACGTTCCTCTTCACTTCCAACAAAGGTGGC p } \\ \text { miRNA-302 } & \\ \text { miRNA-302 A } & \\ \text { miRNA-302 S } & \text { CTAGCTCGAGTGAGAGTGCTGTCCAAGTAAG } \\ \text { muG6PD } & \text { CCGCGGTACCGCTTATGTCTGTAACCGGGTC } \\ \text { G6PD S } & \\ \text { G6PD A } & \text { CCAGATCGGGACCTGCTG } \\ \text { G6PD FL } & \text { AGGCACTCTCTGTAGGCATTCC } \\ \text { G6PD LC } & \text { AGTAGAGAGACCGTGCCCGAG-FL } \\ \end{array}$

of H2O of LightCycler 480 Genotyping Master (Roche), and $2 \mu \mathrm{l}$ cDNA. G6pdh PCR mixture contained $5 \mu$ l G6pdh standard (LightMix Kit G6PDH, TIB MOLBIOL), 5x master mix (LightCycler 480 Genotyping Master, Roche), 16x G6PDH (LightMix Kit human G6PDH, TIB MOLBIOL), 25 mM MgCl 2 (LightCycler 480 Genotyping Master, Roche) and up to $20 \mu$ total volume of $\mathrm{H}_{2} \mathrm{O}$ of LightCycler 480 Genotyping Master (Roche). In all experiments a no template control was used.

For the expression of Kcnh5, KIFC1 and miRNA302, G6pdh was used as a reference gene. Real-time PCR was performed on Light-Cycler 480 II (Roche) with the following parameters: one cycle of preincubation at $95 \mathrm{oC}$ for $10 \mathrm{~min}, 40$ cycles of amplification for KIFC1 and Kcnh5 genes ( $95 \mathrm{oC}$ for $10 \mathrm{sec}, 62^{\circ} \mathrm{C}$ for KIFC1 and $56 \mathrm{oC}$ for Kcnh 5 for $20 \mathrm{sec}$, and $72^{\circ} \mathrm{C}$ for 10 sec), while for G6pdh and miRNA-302 genes 45 cycles of amplification were used $\left(95 \mathrm{oC}\right.$ for $10 \mathrm{sec}, 59^{\circ} \mathrm{C}$ for mG6PD and 60 oC for miRNA-302 for $20 \mathrm{sec}$, and 72 oC for $10 \mathrm{sec}$ ) and one cycle at $4^{\circ} \mathrm{C}$ for cooling. The emitted fluorescence was detected at $640 \mathrm{~nm}$.

Results were obtained as $\mathrm{Cp}$-value and number of copies. Cp-value represents the crossing point at which the sample fluorescence overcomes the background fluorescence. Kcnh5, KIFC1 and miRNA-302 results were normalized to G6pdh ones, based on Müller et $\mathrm{al}^{19}$. Multiple dilutions of G6pdh gene were used in order to obtain a standard curve and to verify the sensitivity of the experiment.

\section{Results}

In order to determine the expression profile of of Kcnh5, KIFC1 and miRNA-302, we divided the collected embryos of every developmental stage at groups of 10 embryos, resulting in 11 speciments for 8-cell stage, 7 for morula stage and 9 for blastocyst stage. The samples underwent RNA extraction and cDNA synthesis and the genes expression in 8-cell, morula and blastocyst stage was studied using quantitative PCR (real-time PCR). The expression results are presented herein as ratios of gene-of- 
interest/G6pdh copies (Figures 1 and 2).

\section{Kcnh5}

Kcnh5 expression profile was investigated in approximately 270 mouse embryos at 8-cell, morula and blastocyst stages, resulting in absence of expression at these developmental stages.

\section{KIFC1}

KIFC1 expression profile was investigated in approximately 270 mouse embryos at 8-cell, morula and blastocyst stages. The expression of this gene was found as positive in all three developmental stages. More specifically, in 8-cell stage the mean detection was at Cp 34.9 (range 31.5-35) with 30.8 copies (range 11.6-74.9), in morula stage at Cp 34.9 (range 34.5-35) with 26.47 copies (range 11.6-45.6) and in blastocyst stage at $\mathrm{Cp} 35$ with 12.9 copies (range 6.74-23). When normalized with G6pdh expression, the Cp ratio was 0.93 (range 0.87-1.07) and the copies ratio 15.06 (range 1.31-64.57) at 8-cell stage. At morula stage, the $\mathrm{Cp}$ ratio was found 1.00 (range 0.86-1.16) and the copies ratio 11.13 (3.3933.53). At blastocyst stage, the $\mathrm{Cp}$ ratio was 1.07 (range 0.88-1.26) and copies ratio 3.91 (range 0.9818.62).

It was found that at 8-cell stage KIFC1 expression is higher than the subsequent developmental stages with a decrease of gene expression up to 3 to 4 fold at the blastocyst stage.

\section{MiRNA-302}

miRNA-302 expression profile was investigated in approximately 270 mouse embryos at 8-cell, morula and blastocyst stages. The expression of this gene was found as positive in all three developmental stages. In 8-cell stage, the mean detection was at $\mathrm{Cp}$ 31.71 (range 29.18-33.59) with 325 copies (range 66.2-392), in morula stage at Cp 31.07 (range 30.1831.58 ) with 385 copies (range 61.5-976) and in blas- tocyst stage at Cp 31.23 (range 30.74-31.53) with 539 copies (range $61.5-976$ ). When normalized with G6pdh expression, the Cp ratio was 0.85 (range 0.790.96 ) and the copies ratio 159.47 (range 1.15-338) at 8-cell stage. At morula stage, the $\mathrm{Cp}$ ratio was found 0.89 (range $0.76-1.04$ ) and the copies ratio 192.28 (10-841). At blastocyst stage, the Cp ratio was 0.95 (range 0.79-1.12) and copies ratio 93.11 (range 16-195).

miRNA-302 expression was detected at all developmental stages and more specifically there was a slight increase of copies from 8-cell to morula stage and subsequently a decrease at blastocyst stage.

\section{Discussion}

Embryonic development is a complicated multifactorial process determined by maternally expressed genes switching to embryonic genes transcription. Microarray technology studies helped to clarify which genes are expressed during these developmental processes. It seems that the 8-cells stage is essential for the activation of embryonic genes transcription at human embryos. It is also known that the genes mainly activated are associated with the cell cycle control. Previously, we had identified several genes over- and underdetected at 8-cell stage blastomeres ${ }^{2}$.

Cell cycle is a complicated process, under the control of several overlapping mechanisms. It is well established that ion channels contribute to cell cycle control via regulation of the transmembrane potential [20]. More specifically, potassium ion channels regulates the resting membrane potential and it is shown that their function is crucial for cell cycle progression since widespectrum potassium channel blockers inhibit proliferation $^{21}$.

Kcnh5 gene encodes Kv10.2 protein (eag2) which belongs to the ether-a-go-go (eag) potassium channel family. This ion channel family participates in several important cellular processes such as regulation of resting membrane potential, cellular volume, proliferation 
and oncogenesis ${ }^{5}$. Kcnh5 channel seems to participate at the early events of mitosis via regulation of cell's volume $^{22}$. Bracey et al showed that Kcnh5 protein binds to alpha and beta-tubulin indicating that this protein might participates in cytoskeleton formation [6]. This finding, in addition to our previous study that showed increased expression at human 8-cell embryonic stage as well as that knockdown of Kcnh5 resulted in inhibition of proliferation, arrest of cells at G1 phase or even triggered apoptosis, indicates that Kcnh5 might play a crucial role in proliferation and more specifically in cytoskeleton formation ${ }^{3,5}$. Therefore, we investigated the expression profile of this protein in three developmental stages of mouse embryos. Surprisingly, there was absence of expression at mouse 8-cell, morula and blastocyst stage. This indicates that although Kcnh5 could contribute in cell cycle control of cell undergoing the usual mitosis process, during blastomere cleavage and blastocyst formation there are other mechanisms of cell cycle control activated independent of potassium channel proteins.

KIFC1 is a member of Kinecin-14 family characterised by minus-end motility on microtubules ${ }^{8}$. It is the only member of this family in mammalian cells ${ }^{23}$. This kinesin is associated with elongation and stabilization of spindle at mitosis and meiosis, biogenesis of acrosome during spermatogenesis and survival of cancer cells with extra centrosomes ${ }^{9,11}$. Additionally, KIFC1 is crucial for bipolar spindle formation and genomic stability ${ }^{9}$. During embryogenesis KIFC1 was found overexpressed in syncytiotrophoblast of normal and pathological human placenta, indicating an active role during implantation ${ }^{12}$. In previous study, Kiessling et al showed that this gene is overexpressed at human 8cell embryos while from our unpublished data it seems that there might be correlation between the levels of KIFC1 expression and morphology score of human 8cell embryos ${ }^{3}$. Therefore, we investigated the expression levels of KIFC1 in three developmental stages of mouse embryos, finding positive expression in all stages. At 8-cell stage, KIFC1/G6pdh ratio counted 15.06 copies, being the highest expression level, at morula stage 11.13 copies and at blastocyst stage 3.91 showing a dramatic approximately 4-fold decrease in KIFC1 expression levels. The increased expression levels of KIFC1 at mouse 8-cell embryos shows that this kinesin might be an important contributor to the early blastomere cleavage process. But when the proliferation turns to a more cell cycle-controlled procedure such as in blastocyst, the KIFC1 expression levels decrease and its role probably diminishes.

miRNA-302 is an important regulator of cell cycle since it was found to contribute to cell cycle regulation of embryonic stem cell (ESC) by targeting cyclin D1 and CDK4 ${ }^{14}$. Additionally, the miRNA 302/367 cluster was found to have a crucial role in G1-S phase transition by promoting proliferation in mouse $\mathrm{ESC}^{17}$. Although, miRNA-302 cluster seems to be an important regulator of cell cycle and is highly expressed in undifferentiated ESCs, there are conflicting data concerning its expression profile during early embryogenesis. Viswanathan et al did not find significant levels of expression at early embryos $^{17}$. On the other hand, Card et al showed that miRNA-302 is expressed at developmental stages E6.5, E7.5 and E8.5. Therefore, we investigated the expression levels of miRNA-302 in three developmental stages, finding positive expression in all of these stages. At 8-cell stage, miRNA-302/G6pdh ratio counted 159.47 copies, at morula stage 192.28 copies, being the highest expression level, and at blastocyst stage 93.11 copies diminished approximately by half. The expression of miRNA-302 during mouse early embryogenesis is at high levels, indicating an important role for the first divisions of blastomeres. Morula is the stage of highest expression levels while there is a decrease by half at blastocyst, indicating that miRNA-302 might play an important role at the events that happen during morula stage. Therefore, it could be useful to identify the events that correlate with highest miRNA-302 expression during morula stage. 
Embryo development in vitro is a long and complicated process, initiation and completion of which involve many factors that act either on the oocyte or the early embryo development. For example, Kcnh5, a potassium channel believed to be crucial for early events of mitosis, was shown to be absent during early embryogenesis, indicating that blastomeres use different mechanisms for division from somatic cells. The gene expression profiles which are associated with embryo developmental competence, clearly demonstrate that there are differences in the expression in each individual stage of the specific genes that we investigate in this study. KIFC1 is believed to play fundamental role in the formation of syncytiotrophoblast of normal and abnormal human placenta involved in the process of implantation. KIFC1 also, has been shown in our study that is present during early embryogenesis with its expression levels decreasing as the embryogenesis progress, indicating a more important role at early events. While miRNA-302 is an important regulator of cell cycle G1/S transition, it was found at highest levels during morula stage indicating that it might participate in other processes except from cell cycle control during early embryogenesis and could play an important role in embryonic control.

We have to take under consideration that these genes are also involved in reprogramming the somatic cells, indicating a possible role in epigenetic reprogramming of the embryos. The challenge now is to correlate the gene function and regulation to specific events in early embryonic development.

\section{Conclusion}

In this study, we investigated the expression profile of Kcnh5, KIFC1 and miRNA-302 at mouse embryos during the developmental transition from 8-cell to blastocyst stage. The Kcnh5 gene was characterized with absence of expression in all three developmental stages while the KIFC1 and miRNA-302 expression was detected in all stages.

\section{Funding}

This research did not receive any specific grant from any funding agency in the public, commercial or not-for-profit sector.

\section{Ethics Aproval}

This study was reviewed and approved by the University and Hospital Ethics Committee and the Animal Care and Use Committee of the Pasteur Institute.

\section{Competing Interests}

The authors declare that they have no competing interests

\section{References}

1. Loutradis D, Drakakis P, Kallianidis K, Milingos S, Dendrinos S and Michalas S: Oocyte morphology correlates with embryo quality and pregnancy rate after intracytoplasmic sperm injection. Fertility and Sterility 1999; 72: 240-4,.

2. Kiessling AA, Bletsa R, Desmarais B, Mara C, Kallianidis $\mathrm{K}$ and Loutradis D: Evidence that human blastomere cleavage is under unique cell cycle control. Journal of assisted reproduction and genetics 2009; 26: 187-95.

3. Kiessling AA, Bletsa R, Desmarais B, Mara C, Kallianidis K and Loutradis D: Genome-wide microarray evidence that 8-cell human blastomeres over-express cell cycle drivers and under-express checkpoints. Journal of assisted reproduction and genetics 2010; 27: 265-76.

4. Vlismas A, Bletsa R, Mavrogianni D, et al: Microarray Analyses Reveal Marked Differences in Growth Factor and Receptor Expression Between 8-Cell Human Embryos and Pluripotent Stem Cells. Stem cells and development 2016; 25: 160-77.

5. Huang X, Dubuc AM, Hashizume R, et al: Voltagegated potassium channel EAG2 controls mitotic entry and tumor growth in medulloblastoma via 
regulating cell volume dynamics. Genes \& development 2012 26: 1780-96.

6. Bracey K, Ju M, Tian C, Stevens L and Wray D: Tubulin as a binding partner of the heag2 voltage-gated potassium channel. The Journal of membrane biology 2008; 222: 115-25.

7. Kocabas AM, Crosby J, Ross PJ, et al: The transcriptome of human oocytes. Proceedings of the National Academy of Sciences of the United States of America 2006; 103: 14027-32.

8. Fink G, Hajdo L, Skowronek KJ, Reuther C, Kasprzak AA and Diez S: The mitotic kinesin-14 Ncd drives directional microtubule-microtubule sliding. Nature cell biology 2009; 11: 717-23.

9. Kim N and Song K: KIFC1 Is Essential for Bipolar Spindle Formation and Genomic Stability in the Primary Human Fibroblast IMR-90 Cell. Cell Structure and Function 2013; 38: 21-30.

10. Yang WX and Sperry AO: C-terminal kinesin motor KIFC1 participates in acrosome biogenesis and vesicle transport. Biology of reproduction 2003; 69: 1719-29.

11. Kwon M, Godinho SA, Chandhok NS, et al: Mechanisms to suppress multipolar divisions in cancer cells with extra centrosomes. Genes \& development 2008; 22: 2189-203.

12. Sati L, Seval-Celik Y, Unek G, Korgun ET and Demir R: The presence of kinesin superfamily motor proteins KIFC1 and KIF17 in normal and pathological human placenta. Placenta 2009; 30: 848-54.

13. Wang Y, Baskerville S, Shenoy A, Babiarz JE, Baehner L and Blelloch R: Embryonic stem cellspecific microRNAs regulate the G1-S transition and promote rapid proliferation. Nature genetics 2008; 40: 1478-83.

14. Wang Y, Melton C, Li YP, et al: miR-294/miR-302 promotes proliferation, suppresses G1-S restriction point, and inhibits ESC differentiation through separable mechanisms. Cell reports
2013; 4: 99-109.

15. Parchem RJ, Moore N, Fish JL, et al: miR-302 Is Required for Timing of Neural Differentiation, Neural Tube Closure, and Embryonic Viability. Cell reports 2015; 12: 760-73.

16. Lin SL, Chang DC, Lin CH, Ying SY, Leu D and Wu DT: Regulation of somatic cell reprogramming through inducible mir-302 expression. Nucleic acids research 2011; 39: 1054-65.

17. Viswanathan SR, Mermel CH, Lu J, Lu CW, Golub TR and Daley GQ: microRNA expression during trophectoderm specification. PloS one 2009; 4: e6143.

18. Card DA, Hebbar PB, Li L, et al: Oct4/Sox2-regulated miR-302 targets cyclin D1 in human embryonic stem cells. Molecular and cellular biology 2008; 28: 6426-38.

19. Muller MC, Saglio G, Lin F, et al: An international study to standardize the detection and quantitation of BCR-ABL transcripts from stabilized peripheral blood preparations by quantitative RT-PCR. Haematologica 2007; 92: 970-73.

20. Blackiston DJ, McLaughlin KA and Levin M: Bioelectric controls of cell proliferation: ion channels, membrane voltage and the cell cycle. Cell cycle 2009; 8: 3527-36.

21. DeCoursey TE, Chandy KG, Gupta S and Cahalan MD: Voltage-gated $\mathrm{K}+$ channels in human $\mathrm{T}$ lymphocytes: a role in mitogenesis? Nature 1984; 307: 465-8.

22. Huang $X$ and Jan LY: Targeting potassium channels in cancer. The Journal of cell biology 2014; 206: 151-62.

23. Dagenbach EM and Endow SA: A new kinesin tree. Journal of cell science 2004; 117: 3-7.

Received 28-3-2019

Revised 22-4-2019

Accepted 3-5-2019 